\title{
Prevalência de nódulos pulpares em molares de estudantes de Odontologia e fatores associados
}

\author{
Prevalence of pulp nodules in Dental students' molar teeth \\ and associated factors
}

\author{
Maicon Selayaran* \\ Raquel Padão Guerra* \\ Felipe Brunatto Luz** \\ Fernanda Geraldo Pappen ${ }^{* * *}$ \\ Ana Paula Neutzling Gomes ${ }^{* * *}$
}

\section{Resumo}

Objetivo: avaliar a prevalência de nódulos pulpares em acadêmicos de Odontologia da Universidade Federal de Pelotas, procurando estabelecer associações entre nódulos pulpares e fatores como cárie dentária, restaurações, tratamento ortodôntico prévio e bruxismo. Métodos: a história dentária assim como a de hábitos bucais relacionados ao diagnóstico de bruxismo foram investigadas por meio de entrevista individual. Os participantes foram submetidos a exame clínico e radiográfico, sendo realizadas duas radiografias interproximais na região dos molares, onde foi calculada a frequência de cálculos pulpares nos indivíduos. A associação entre fatores como sexo, idade, restaurações, cárie, tratamento ortodôntico e bruxismo com a presença de nódulos foi calculada pelos testes qui-quadrado e correlação de Spearman $(p<0,05)$. Resultados: Participaram da pesquisa 101 estudantes de Odontologia, com média de idade de 22,75 anos. Do total, 69,3\% apresentaram nódulos pulpares. Dentre os 762 dentes avaliados, 29,5\% apresentaram nódulos pulpares. As calcificações foram mais frequentes nos primeiros molares. Um total de 45,5\% dos alunos recebeu diagnóstico compatível com bruxismo. Fatores como bruxismo, tratamento ortodôntico prévio e cárie dentária não tiveram associação com a ocorrência de nódulos pulpares $(p>0,05)$. Os resultados demonstraram haver associação dos nódulos pulpares apenas com a presença de restaurações ( $p=$ 0,009). Conclusões: É possível concluir que a prevalência de nódulos pulpares é alta na população estudada, estando relacionada, principalmente, com a presença de restaurações.

Palavras-chave: Calcificações da polpa dentária. Radiografia interproximal. Bruxismo.

\section{Introdução}

As calcificações pulpares surgem como corpos livres no tecido pulpar, mas podem se encontrar aderidas ou embutidas nas paredes dentinárias. Essas calcificações podem ser divididas em tipos distintos: dentículos, compostos por restos epiteliais circundados por dentina secretada por odontoblastos; calcificações lineares difusas, que compreendem pequenas áreas calcificadas irregulares e paralelas aos vasos sanguíneos e feixes nervosos da polpa; e cálculos pulpares, que seguem um padrão concêntrico de calcificação em torno de uma área de células necróticas ou fibras colágenas ${ }^{1}$.

As calcificações pulpares são mais frequentes na polpa coronária de dentes posteriores, sendo os pré-molares menos acometidos que os molares. Somente calcificações com mais de $200 \mu$ m de diâmetro são visíveis radiograficamente, e um ou mais cálculos pulpares podem ser encontrados em um mesmo dente ${ }^{2}$.

Com relação à etiologia, sabe-se que a formação de calcificações pulpares ainda não foi completamente esclarecida, e diversos fatores causais vêm sendo relacionados com a sua ocorrência. Sabe-se que idade ${ }^{3,4}$, distúrbios circulatórios na polpa ${ }^{5}$ e predisposição genética têm relação com o processo ${ }^{2,6}$. Existem, ainda, anomalias congênitas relacionadas com a formação de calcificações pulpares, como a displasia dentinária tipo $\mathrm{II}^{7}$ e as síndromes de Marfan e de Ehlers-Danlos ${ }^{8}$.

Acadêmicos de Odontologia, Faculdade de Odontologia da Universidade Federal de Pelotas, Pelotas, RS, Brasil.

Mestrando, Área de Diagnóstico Bucal, Faculdade de Odontologia da Universidade Federal de Pelotas, Pelotas, RS, Brasil.

Professora Adjunta, Departamento de Semiologia e Clínica, Faculdade de Odontologia da Universidade Federal de Pelotas, Pelotas, RS, Brasil. 
Também, lesões cariosas, restaurações profundas e abrasão são, frequentemente, descritas como possíveis fatores causais das calcificações pulpares $^{4,9,10}$. A progressão da cárie e a presença de restaurações profundas induzem a formação de dentina terciária com a função de proteger o tecido pulpar da agressão cariosa. Em lesões muito extensas e profundas, pode haver uma grande deposição dessa dentina na cavidade pulpar, ou a lesão de cárie pode induzir as células da polpa a produzirem focos de dentina no interior do tecido, podendo acarretar no aparecimento de calcificações pulpares capazes até de causar a obliteração da câmara ${ }^{11}$.

Uma possível ruptura, mesmo que parcial, do suprimento vascular apical da polpa, após traumatismos alvéolo-dentários, pode gerar uma isquemia transitória ou a formação de um coágulo no tecido pulpar, com consequente depósito de tecido calcificado em decorrência da estimulação dos odontoblastos ${ }^{12-14}$. Da mesma forma que os traumatismos, porém com menor frequência, o tratamento ortodôntico representa estresse adicional para o tecido pulpar, que, numa tentativa de defesa, induziria a formação de calcificações ${ }^{2,8,15-17}$.

Apesar de não haver relatos na literatura relacionando a ocorrência de bruxismo com calcificações pulpares, esse hábito também poderia representar um estímulo para a sua formação. $\mathrm{O}$ apertamento dentário consiste numa atividade inconsciente de apertar e ranger os dentes de forma rítmica ou espasmódica durante o sono e tem a capacidade de provocar dor facial e desgaste dentário ${ }^{18}$.

Tendo em vista os inconvenientes que a ocorrência de nódulos pulpares acarretam ao tratamento endodôntico, dificultando e muitas vezes impedindo $o$ acesso aos canais radiculare ${ }^{19}$, torna-se clara a necessidade de mais estudos que investiguem a etiologia das calcificações, bem como sua prevalência. O objetivo deste estudo foi avaliar a prevalência de nódulos pulpares na câmara pulpar de molares de estudantes de Odontologia e fatores associados.

\section{Métodos}

O presente estudo teve parecer favorável à sua execução (№ 114/2009) pelo Comitê de Ética em Pesquisa Institucional. A prevalência de nódulos pulpares foi investigada por meio de exames radiográficos, buscando relacionar essa ocorrência com fatores causais como cáries, restaurações, bruxismo e tratamento ortodôntico, bem como idade e sexo. A inclusão desses participantes ocorreu de forma voluntária mediante convite aos 450 alunos da Faculdade de Odontologia, durante o ano de 2010. No total, 101 alunos aceitaram participar do estudo e assinaram um termo de consentimento livre e esclarecido anteriormente à realização dos exames.
Para cada indivíduo, foi realizada uma anamnese, com coleta de informações a respeito do diagnóstico de bruxismo, restaurações e tratamento ortodôntico prévio. Durante o exame clínico, foi avaliada a presença de facetas de desgaste nos dentes, tanto anteriores como posteriores, presença de cárie, presença de restaurações e hipertrofia do músculo masseter, a qual foi considerada presente quando houvesse um aumento de volume na região do músculo ao solicitar que o voluntário ocluísse com força.

Foram diagnosticados como bruxômanos os pacientes que apresentaram facetas de desgastes alinhadas em dentes anteriores e/ou posteriores, além da presença atual de pelo menos um dos seguintes sinais e sintomas de bruxismo: autorrelato de ranger os dentes durante o sono e/ou vigília, sensibilidade dolorosa à palpação nos músculos mastigatórios masseter e/ou temporal e desconforto na musculatura ao despertar ${ }^{20}$.

A avaliação da presença de nódulos foi realizada nos primeiros e segundos molares, superiores e inferiores, de cada indivíduo, por meio de exame radiográfico. Para cada participante foram feitas duas tomadas radiográficas interproximais da região de molares (direita e esquerda), com tempo de exposição adequado para cada paciente e uso de avental de chumbo para proteção do indivíduo exposto aos raios $\mathrm{X}$, em um aparelho operando com $60 \mathrm{kVp}$ e 7 a $10 \mathrm{~mA}$, (Spectro 70x, Dabi Atlante ${ }^{\circledR}$, Ribeirão Preto, SP). Foram utilizadas películas radiográficas de tamanho standard e sensibilidade E (Kodak Dental $^{\circledR}$, Radiography Series, Rio de Janeiro, RJ) e posicionador interproximal. $\mathrm{O}$ processamento das radiografias foi feito em câmara escura, com tempos de revelação e fixação padronizados, seguindo o método temperatura/tempo (Kodak Dental ${ }^{\circledR}$, Radiography Series, Rio de Janeiro, RJ).

As radiografias foram analisadas por dois examinadores previamente calibrados, e com índice de concordância Kappa $=0,8$. A avaliação das radiografias foi realizada em sala escura, utilizando negatoscópio (VH Dental Equipments ${ }^{\circledR}$, Araraquara, $\mathrm{SP})$ e lupa com $2 \mathrm{X}$ de aumento. Durante a análise, cada dente foi classificado quanto à presença de nódulos pulpares (ausente ou presente), lesão de cárie (ausente ou presente) e restauração (ausente ou presente). Os nódulos pulpares foram identificados como imagens radiopacas presentes no interior da câmara pulpar, independentemente do tamanho.

Foram considerados como critérios para exclusão do estudo: ausência de dente, dente tratado endodonticamente, dentes com bandas ortodônticas que impossibilitassem a visualização total da câmara pulpar e dentes com giroversão acentuada que impossibilitasse a captura, sem distorções, da câmara pulpar na tomada radiográfica.

Foram incluídos no estudo 762 dentes. Quarenta e seis dentes foram excluídos do estudo, pois se apresentavam com tratamento endodôntico, com bandas ortodônticas que impossibilitaram a visua- 
lização total da câmara pulpar ou giroversão acentuada que impossibilitava a captura da câmara pulpar na tomada radiográfica.

Os dados foram submetidos à análise estatística no programa SigmaStat ${ }^{\circledR}$ versão 3.5 for Windows. Foi calculada a frequência de distribuição dos cálculos pulpares nos indivíduos e investigada a associação entre fatores como sexo, idade, restaurações, cárie, tratamento ortodôntico e bruxismo com a presença de nódulos, por meio dos testes qui-quadrado e correlação de Spearman $(p<0,05)$.

\section{Resultados}

Do total da amostra, 70 estudantes apresentaram nódulos pulpares (69,3\%). A média de idade dos 101 estudantes que participaram da pesquisa foi de $22,75$ ( $\pm 2,809)$ anos. Não houve correlação entre idade e ocorrência de nódulos pulpares $(\mathrm{p}=0,158)$.

Do total da amostra, 46 (45,5\%) alunos receberam diagnóstico compatível com bruxismo. Setenta $(69,3 \%)$ estudantes relataram ter feito tratamento ortodôntico prévio, com tempo médio de duração de $42,1( \pm 28,53)$ meses.

Dos 762 dentes avaliados, 225 (29,5\%) apresentaram nódulos pulpares. Dentre os primeiros molares, 154 (41,5\%) apresentaram nódulos pulpares, tendo sido observados nódulos pulpares em 71 $(18,2 \%)$ segundos molares $(p=0,000)$. Os resultados demonstraram haver associação entre a presença de restaurações e o aparecimento de nódulos pulpares ( $\mathrm{p}=0,009$ ), porém, não foi observada associação entre a presença de lesões de cárie $(\mathrm{p}=0,757)$ e a ocorrência de nódulos.

As associações entre a presença de nódulos pulpares e fatores como sexo, diagnóstico de bruxismo e tratamento ortodôntico prévio, cárie e restaurações estão descritas na Tabela 1.

Tabela 1- Associação entre a ocorrência de nódulos pulpares e as variáveis estudadas, em 762 molares de 101 estudantes de Odontologia da Universidade Federal de Pelotas

\begin{tabular}{l|r|r|r}
\hline \multicolumn{1}{c|}{ Variáveis } & \multicolumn{1}{c|}{$\mathrm{n}(\%)$} & \multicolumn{1}{c}{$\begin{array}{c}\text { Nódulos } \\
\text { pulpares }(\%)\end{array}$} & Valor de P \\
\hline Sexo & $101(100))$ & & \\
Feminino & $63(62,4)$ & $42(66,7)$ & 0,459 \\
Masculino & $38(37,6)$ & $28(73,7)$ & \\
Bruxismo & $101(100)$ & & \\
Ausente & $55(54,5)$ & $37(67,3)$ & 0,628 \\
Presente & $46(45,5)$ & $33(71,7)$ & \\
Tratamento ortodôntico & $101(100)$ & & 0,239 \\
Ausente & $31(30,7)$ & $24(77,4)$ & 0,000 \\
Presente & $70(69,3)$ & $46(65,7)$ & \\
Dente & $762(100)$ & & \\
Primeiro molar & $371(48,7)$ & $154(41,5)$ & 0,506 \\
Segundo molar & $391(51,3)$ & $71(18,2)$ & \\
Lesão de cárie & $762(100)$ & & \\
Ausente & $752(98,7)$ & $223(29,7)$ & 0,005 \\
Presente & $10(1,3)$ & $2(20)$ & \\
Restauração & $762(100)$ & & \\
Ausente & $487(63,9)$ & $127(26,1)$ & \\
Presente & $275(36,1)$ & $98(35,6)$ & \\
\hline
\end{tabular}

"Teste qui-quadrado.

\section{Discussão}

A ocorrência de nódulos pulpares foi observada em 70 estudantes examinados (69,3\%). Dependendo da população estudada, da faixa etária dos indivíduos examinados, dos grupos dentários examinados e do método utilizado, a prevalência de nódulos pulpares é bastante variada na literatura. O índice que encontramos é mais elevado que o relatado na maioria dos estudos, que mostram uma prevalência entre $38 \%$ e 51,4\% $\%^{2,4,21-24}$. Quando considerados os possíveis fatores associados, dados da literatura apontam para uma maior prevalência de nódulos quanto maior a idade do indivíduo ${ }^{3,4}$. No entanto, no presente estudo, não foi encontrada associação entre idade e o aparecimento de nódulos pulpares $(\mathrm{p}=0,050)$, possivelmente pelo fato de nossa população ser constituída basicamente de indivíduos jovens, com uma média de idade de 22,75 anos.

Baghdady et al. ${ }^{25}$ (1998), assim como Ranjitkar et al. $^{2}$ (2002), relataram não haver associação entre gênero e a ocorrência de nódulos pulpares. Resultados semelhantes foram encontrados em nosso estudo, onde $66,7 \%$ das mulheres e $73,6 \%$ dos homens apresentaram nódulos, não havendo associação entre as variáveis $(\mathrm{p}=0,898)$.

Dos 762 dentes que atenderam os critérios para inclusão na amostra, 225 apresentaram nódulos, sendo estes mais prevalentes nos primeiros molares do que nos segundos molares. A maior frequência de nódulos pulpares nos primeiros molares em relação aos segundos molares já vem sendo relatada na literatura ${ }^{2,23,26,27}$. Provavelmente, os primeiros molares, por irromperem primeiro, encontram-se por um período mais longo expostos aos fatores relacionados ao desenvolvimento dos nódulos pulpares. Além disso, esses dentes possuem um tecido pulpar mais volumoso que requer maior suprimento sanguíneo, o que pode favorecer a formação de nódulos ${ }^{27}$.

Neste trabalho, procuramos verificar a associação entre tratamento ortodôntico prévio e a presença de nódulos pulpares, uma vez que o tratamento ortodôntico poderia representar estresse adicional para o tecido pulpar, que, numa tentativa de defesa, induziria a formação de nódulos. Entretanto, não foi encontrada essa associação $(p=0,346)$. Corroborando o presente resultado, estudos vêm demonstrando que a polpa dentária não envelhece estruturalmente pela aplicação de um tratamento ortodôntico e que o envelhecimento pulpar também não revela relação direta ou indireta com a idade cronológica do paciente, mas está diretamente relacionado com fatores ambientais como atrição, abrasão, erosão, abfração, traumatismos, cáries e procedimentos restauradores ${ }^{26,28}$.

Alguns estudos apontam a cárie dentária como um dos possíveis fatores de formação de nódulos pulpares, pois o processo carioso induz a formação de dentina terciária, para que esta possa proteger a 
polpa dentária dos produtos tóxicos produzidos pelas bactérias cariogênicas. Ainda, esses mesmos produtos tóxicos podem desencadear um processo de irritação crônica ao tecido pulpar, que reage com a formação de nódulos pulpares ${ }^{4,5,9}$. No presente estudo, não foi possível verificar associação entre a presença de cárie dentária e nódulos pulpares ( $\mathrm{p}=0,757)$, resultado que se deve, provavelmente, ao baixo índice de lesões de cárie encontrado.

No entanto, nosso estudo demonstrou haver associação entre a presença de restaurações e a ocorrência de nódulos pulpares $(\mathrm{p}=0,009)$. A presença de restaurações é considerada um possível fator para 0 surgimento de nódulos pulpares, na medida em que o procedimento operatório, restaurador e o material utilizado podem ser irritantes para a polpa, estimulando o surgimento desses nódulos ${ }^{4,5,9}$.

Uma das hipóteses do presente estudo foi a de que o bruxismo, por causar estresse oclusal e desgaste dentário, levaria à irritação do tecido pulpar, podendo culminar com a indução da formação de nódulos. No entanto, não houve associação entre a presença de nódulos pulpares e o diagnóstico de bruxismo, pois a frequência de nódulos pulpares em indivíduos bruxômanos foi próxima à frequência encontrada em indivíduos não bruxômanos ( $\mathrm{p}=0,789)$.

Apesar da existência de técnicas que permitem que, na maioria dos casos, as dificuldades oferecidas durante a realização do tratamento endodôntico de dentes com calcificação pulpar sejam contornadas, os nódulos podem gerar dificuldades clínicas ou até impedir o acesso aos canais radiculares ${ }^{29-31}$, o que torna importante o estudo da sua etiologia e prevalência na população.

\section{Conclusão}

De acordo com os resultados obtidos, a prevalência de nódulos pulpares é alta na população estudada, estando relacionada, principalmente, com a presença de restaurações.

\section{Abstract}

Objective: this study evaluated the prevalence of pulp nodules in dental students from the Federal University of Pelotas. The study also tried to establish associations between pulp nodules and elements such as caries, restorations, previous orthodontic treatment, and bruxism. Methods: an individual interview was applied to investigate the dental history and oral habits related to bruxism diagnosis. Every participant was submitted to clinical and radiographic examination. Two bitewing radiographs in the molar area were performed in each student and the frequency of pulp stones was assessed. The association between factors such as gender, age, restorations, caries, orthodontic treatment, and bruxism with the presence of nodules was assessed by the chisquare test and Spearman's correlation coefficient ( $p<$
0.05). Results: a total of 101 dental students participated in the research. The age average of the participants was 22.75 years old. From the total of students, $69.3 \%$ presented pulp nodules. Considering the 762 teeth studied, $29.5 \%$ presented pulp nodules. Calcifications were more frequent in first molar teeth. A total of $45.5 \%$ of students were diagnosed with bruxism. There was no association between the occurrence of pulp stones and the following factors: bruxism, orthodontic treatment, and caries $(p>0.05)$. The results showed an association only between pulp nodules and restorative procedures $(p=0.009)$. Conclusions: it is possible to conclude that the prevalence of pulp nodules in the studied population is high, and it is mainly related to restorative procedures.

Keywords: Dental pulp calcification. Bitewing radiography. Bruxism.

\section{Referências}

1. Neville BW, Damm DD, Allem CM, Bouquot JE. Patologia oral e maxilofacial. 3. ed. Philadelphia: Elsevier; 2009.

2. Ranjitkar S, Taylor JA, Townsend GC. A radiographic assessment of the prevalence of pulp stones in Australians. Aust Dent J 2002; 47(1):36-40.

3. Hillmann G, Geurtsen W. Light-microscopical investigation of the distribution of extracellular matrix molecules and calcifications in human dental pulps of various ages. Cell Tissue Res 1997; 289(1):145-54.

4. Sayegh FS, Reed AJ. Calcification in the dental pulp. Oral Surg 1968; 25(6):873-82.

5. Sundell JR, Stanley HR, White CL. The relationship of coronal pulp stone formation to experimental operative procedures. Oral Surg 1968; 25(4):579-89.

6. Van Den Berghe JM, Panther B, Gound TG. Pulp stones throughout the dentition of monozygotic twins. Oral Surg Oral Med Oral Pathol Oral Radiol Endod 1999; 87(6): 749-51.

7. Goga R, Chandler NP, Oginni AO. Pulp stones: a review. Int Endod J 2008; 41(6):457-68.

8. Bauss O, Röhling J, Rahman A, Kiliaridis S. The effect of pulp obliteration on pulpal vitality of orthodontically intruded traumatized teeth. J Endod 2008; 34(4):417-20.

9. Arys A, Philippart C, Dourov N. Microradiography and light microscopy of mineralization in the pulp of undemineralized human primary molars. J Oral Pathol Med 1993; 22(2): 49-53.

10. Gibilisco JA. Stafne's oral radiographic diagnosis. 5. ed. Philadelphia: WB Saunders; 1985.

11. Katchburian E, Arana V. Histologia e embriologia oral. 1. ed. São Paulo: Panamericana; 1999.

12. Soporowski N, Allred E, Needleman H. Luxation injuries of primary anterior teeth: prognosis and related correlates. Pediatr Dent 1994; 16:96-101.

13. Andreasen JO, Andreasen FM. Texto e atlas colorido de traumatismo dental. Porto Alegre: Artmed; 2001.

14. Choi SC, Park JH, Pae A, Kim JR. Retrospective study on traumatic dental injuries in preschool children at Kyung Hee Dental Hospital, Seoul, South Korea. Dent Traumatol 2010; 26(1):70-5. 
15. Delivanis HP, Sauer GJR. Incidence of canal calcification in the orthodontic patient. Am J Orthod 1982; 82(1):58-61.

16. Woloshyn H, Årtun J, Kennedy DB, Joondeph DR. Pulpal and periodontal reactions to orthodontic alignment of palatally impacted canines. Angle Orthod 1994; 64(4):257-26.

17. Stenvik A, Mjör IA. Epithelial remnants and denticle formation in the human dental pulp. Acta Odontol Scand 1970; 28(5):72-8.

18. Branco RS, Branco CS, Tesch RS, Rapoport A. Frequência de relato de parafunções nos sub-grupos diagnósticos de DTM de acordo com os critérios diagnósticos para pesquisa em disfunções temporomandibulares (RDC/TMD). R Dent Press Ortodon Ortop Facial 2008; 13(2):61-9.

19. Ibarrola JL, Knowles KI, Ludlow MO, Mckinley IBJR. Factors affecting the negotiability of second mesiobuccal canals in maxillary molars. J Endod 1997; 23(4):236-8.

20. Carvalho ALA, Del Bel-Cury AA, Rodrigues-Garcia RCM. Prevalence and association of bruxism and emotional stress in Brazilian police officers. Braz Oral Res 2008; 22(1):31-5.

21. Sener S, Cobankara FK, Akgünlü F. Calcifications of the pulp chamber: prevalence and implicated factors. Clin Oral Investig 2009; 13(2):209-15.

22. Tamse A, Kaffe I, Littner MM, Shani R. Statistical evaluation of radiologic survey of pulp stones. J Endod 1982; $8(1): 455-8$.

23. Hamasha AA-H, Darwazeh A. Prevalence of pulp stones in Jordanian adults. Oral Surg Oral Med Oral Pathol Oral Radiol Endod 1998; 86(1):730-2.

24. Chandler NP, Pitt Ford TR, Monteith BD. Coronal pulp size in molars: a study of bitewing radiographs. Int Endod $\mathrm{J}$ 2003; 36(1):757-63.

25. Baghdady V, Leonora B, Ghose J, Huda Y. Prevalence of pulp stones in a teenage Iraqi group. J Endod 1988; 14(6):309-11.

26. Al-Nazhan S, Al-Shammrani S. Prevalence of pulp stones in saudi adults. Prosthodontics 1991; 16(1):129-42.

27. Herrera M, Castro J. Prevalência de nódulos pulpares. Rev ADM 2001; 8(4):130-7.

28. Consolaro CA, Bernardini VR. Metamorfose cálcica da polpa e necrose pulpar asséptica no planejamento ortodôntico. $\mathrm{R}$ Dental Press Ortodon Ortop Facial 2007; 12(6):21-3.

29. Pashley DH, Walton RE, Slavkin HC. Histology and physiology of the dental pulp. In: Ingle JI, Bakland LK. Endodontics. 5. ed. Hamilton: BC Decker; 2002. p. 43-5.

30. Stamos DG, Haasch GC, Chenail B, Gerstein H. Endosonics: clinical impressions. J Endod 1985; 11(4):181-7.

31. Pitt Ford TR, Rhodes JS, Pitt Ford HE. Endodontics problem-solving in clinical practice. London: Martin Dunitz; 2002 .
Endereço para correspondência:

Fernanda Geraldo Pappen

Faculdade de Odontologia - Universidade

Federal de Pelotas

Rua Gonçalves Chaves 457, sala 604

96015-560 Pelotas-RS

Fone: +55 5332256741

E-mail: ferpappen@yahoo.com.br

Recebido: 11/07/2012. Aceito: 15/04/2013. 